\title{
Avaliação da técnica de ELISA para pesquisa de IgG anti-Toxoplasma gondii em exsudatos de carnes de sol
}

\author{
Evaluation of the ELISA technique to search for anti-Toxoplasma gondii IgG in juices \\ from sun dried meat
}

\author{
Maria Aparecida Moraes Marciano* (1), Heitor Franco de Andrade Junior², Luciana Regina Meireles² \\ 1 Instituto Adolfo Lutz (IAL), Centro de Alimentos, Núcleo de Morfologia e Microscopia, São Paulo/SP - Brasil \\ 2 Universidade de São Paulo (USP), Laboratório de Protozoologia, Instituto de Medicina Tropical de São Paulo, São Paulo/SP - Brasil
}

\section{${ }^{*}$ Corresponding Author}

Maria Aparecida Moraes Marciano, Instituto Adolfo Lutz (IAL), Centro de Alimentos, Núcleo de Morfologia e Microscopia, Av. Dr. Arnaldo, 355, Cerqueira César, CEP: 01246-902, São Paulo/SP - Brasil, e-mail: mamarciano@usp.br

Cite as: Evaluation of the ELISA technique to search for anti-Toxoplasma gondii IgG in juices from sun dried meat. Braz. J. Food Technol., v. 21, e2017009, 2018

\section{Resumo}

Toxoplasmose é uma importante zoonose de veiculação hídrica e alimentar, cujo agente é o Toxoplasma gondii. A toxoplasmose acomete seres humanos e animais de produção. É uma zoonose cosmopolita e sua prevalência está relacionada ao consumo de carne crua ou mal cozida, sendo este hábito considerado um dos principais fatores de risco. O controle do potencial de transmissão da toxoplasmose pela carne é relevante, principalmente em produtos artesanais, como as carnes de sol, que são processadas artesanalmente e podem ser consumidas cruas ou mal passadas. Neste estudo, propomos a avaliação da presença de imunoglobulinas da classe IgG anti- $T$. gondii pela técnica de imunoensaio ELISA, padronizando uma diluição do exsudato cárneo obtido pelo descongelamento das carnes de sol produzidas a partir de músculos de animais experimentais e também de carnes de sol comercializadas em "Casas do Norte". Nossos resultados demonstram que o potencial do exsudato cárneo como um material biológico é limitado em carnes processadas, possibilitando o ensaio somente das amostras que obtiveram uma absorbância em densidade óptica (DO) igual ou superior a 0,116, faixa que garante a presença de sangue suficiente para o ensaio da ELISA, permitindo aferir imunoglobulinas específicas anti-T. gondii. Dentre as 32 amostras de carne de sol obtidas de cortes de bovinos experimentais, somente nove $(28 \%)$ apresentaram sangue suficiente para ser ensaiadas, mensuradas pela DO igual ou superior a 0,116 , sendo sete positivas, obtidas de cortes de animais infectados, e duas negativas, confeccionadas com cortes de animais controle. Dentre as 42 amostras de exsudatos de carne de sol obtidas no varejo, somente seis $(14,28 \%)$ apresentaram sangue suficiente para serem ensaiadas e somente uma apresentou imunoglobulinas anti-T. gondii, concordante com dados da literatura. Nossos resultados são promissores e demonstram a importância do desenvolvimento de pesquisa e novas abordagens no controle sanitário dos alimentos ofertados à população.

Palavras-chave: Toxoplasmose; Doença transmitida por alimentos; Carne de sol; Zoonose; Exsudato cárneo; Segurança alimentar.

\section{Abstract}

Toxoplasmosis is an important waterborne and foodborne zoonosis, whose agent is Toxoplasma gondii, and it affects both humans and production animals. This zoonosis is cosmopolitan and its prevalence is related to the consumption of raw or undercooked meat, this habit being considered one of the main risk factors. The control of the potential transmission of toxoplasmosis by meat is relevant, especially in artisanal products such as sun dried meats, which are handcrafted and may be consumed raw or undercooked. In this study, we proposed to evaluate the presence of immunoglobulins of the IgG anti T. gondii class by an ELISA immunoassay technique, standardizing a dilution of the meat juice obtained by defrosting sun dried meat produced from the muscles of experimentally infected animals and also from sun dried meats commercialized in 
shops in the north of Brazil. The results showed that the potential of the meat juice as a biological material was limited in processed meats, and the test was only possible in samples that showed an optical density (OD) equal to or greater than 0.116, a range that guaranteed the presence of sufficient blood for the ELISA assay, allowing for the measurement of specific anti-T. gondii immunoglobulins. Of the 32 samples of beef obtained from cuts from experimental cattle, only $9(28 \%)$ presented sufficient blood to be tested, as measured by an OD equal to or greater than $0.116,7$ being positive, obtained from cuts from infected experimental animals, and 2 negative, obtained from cuts from control animals. Of the 42 samples of juice from retail sun dried meat, only 6 (14.28\%) presented enough blood for the tests and only one presented anti T. gondii immunoglobulins, in agreement with data from the literature. The results were promising and demonstrated the importance of developing research and new approaches in the sanitary control of food offered to the population.

Keywords: Toxoplasmosis; Foodborne diseases; Sun-dried beef; Zoonosis; Meat juice; Food safety.

\section{Introdução}

A toxoplasmose é uma das principais zoonoses mundiais transmitidas por alimentos, principalmente pela ingestão de carne crua ou mal cozida (TENTER, 2009). Seu agente etiológico, Toxoplasma gondii, é um protozoário intracelular que acomete o ser humano e os animais de produção e selvagens (DUBEY; THULLIEZ, 1993); entretanto, seu ciclo sexuado ocorre somente nos felinos, que são seus hospedeiros definitivos (FRENKEL et al., 1970). Estes animais liberam oocistos no ambiente por meio de suas fezes, contaminando o solo e a água, e infectando o homem e os animais de produção.

O controle das doenças de transmissão alimentar em especial, das parasitoses, como a toxoplasmose - tem sido objeto de discussão na área de Saúde Pública, já que a prevalência de infecção por T. gondii está diretamente relacionada às condições sanitárias e aos hábitos alimentares da população (NARDI et al., 2012). O consumo de carne crua ou mal cozida é um dos aspectos mais relevantes na transmissão da toxoplasmose (TENTER, 2009), enfatizando a importância do controle da qualidade da carne e dos seus subprodutos. Os cistos teciduais presentes na carne são relativamente resistentes às mudanças de temperatura e permanecem infectantes em carcaças refrigeradas $\left(-1^{\circ} \mathrm{C}\right.$ a $\left.-4{ }^{\circ} \mathrm{C}\right)$ ou em carne moída, por até três semanas, nesta temperatura (DUBEY; KIRKBRIDE, 1989; DUBEY et al., 1990). Esse período é geralmente maior que o tempo que a carne permanece viável para consumo, ou seja, o tempo em que ela é comercializada.

Estudos europeus relatam a ingestão de carne crua bovina como fator de risco para aquisição da infecção pelo T. gondii (BARIL et al., 1999; COOK et al., 2000) e, infelizmente, no Brasil, ainda não estão disponíveis, no mercado, carnes controladas quanto ao risco de ingestão de cistos de Toxoplasma gondii. Grupos de risco, como imunossuprimidos, idosos, crianças e gestantes, poderiam evitar o consumo de carnes e outros alimentos contaminados com formas infectantes do agente, se métodos de diagnósticos sensíveis e rápidos estivessem disponíveis para aplicação em larga escala na linha de abate, ou mesmo em amostras de carne já processadas para comercialização (VILLENA et al., 2012).

O exame direto da carne, por PCR ou bioensaio, para pesquisa de cistos de T. gondii, não é suficiente para garantir sua segurança, uma vez que os cistos não apresentam uma distribuição homogênea na carne (MECCA et al., 2011). A inativação dos cistos de T. gondii presentes em produtos cárneos pode ser realizada por aquecimento à temperatura superior a $67^{\circ} \mathrm{C}$ (DUBEY et al., 1990) ou por congelamento, a $-13^{\circ} \mathrm{C}$, desde que atinja o centro geométrico da carne (KOTULA et al., 1991). O processamento por cura de alguns produtos cárneos pode inviabilizar os cistos, mas depende da concentração de sal, da temperatura e do tempo de estocagem. Experimentalmente, os cistos foram inativados em solução de $6 \%$ de $\mathrm{NaCl}$ entre $4{ }^{\circ} \mathrm{C}$ e $20{ }^{\circ} \mathrm{C}$, mas resistem por semanas em soluções de $0,85 \%$ a 3,3\% de $\mathrm{NaCl}$, nas mesmas temperaturas (DUBEY, 1997). Também foi demonstrado que a salga não inativa cistos em linguiças suínas caseiras tipo frescal (JAMRA et al., 1991); contudo, em altas concentrações de $\mathrm{NaCl}$, é possível a inativação dos cistos, mas as características organolépticas da carne são alteradas (NAVARRO et al., 1992).

Atualmente, não há um programa nacional de controle sanitário para detecção de cistos de T. gondii na carne destinada ao consumo humano. O controle da toxoplasmose, por meio dos serviços de Inspeção Sanitária na linha de abate, é inviável, já que o cisto de T. gondii é microscópico e, portanto, não pode ser visualizado por exame macroscópico que é utilizado na inspeção, diferentemente de outras zoonoses transmitidas via carne, como a teníase.

Os surtos de toxoplasmose atribuídos à ingestão de cistos presentes na carne crua ou mal passada, no Brasil, são esporádicos e sua baixa notificação pode estar relacionada ao fato de ser considerada uma zoonose de notificação compulsória (DIAS; FREIRE, 2005).

Vale ressaltar a importância de se monitorar a carne de sol frente à toxoplasmose, uma vez que a mesma é elaborada sem padrões oficiais de identidade e qualidade, e, muitas vezes, em condições sanitárias 
inadequadas, representando um problema importante de Saúde Pública. Além disso, a comercialização do produto é facilitada pelo fato de dispensar armazenamento sob refrigeração (MENUCCI et al., 2010). No presente estudo, propomos a padronização de um ensaio imunoenzimático ELISA, utilizando exsudato cárneo bovino, proveniente de amostras de carne de sol. Esta abordagem é factível de execução em nosso meio, permitindo a automação, em larga escala, para avaliação da qualidade sanitária desse alimento, que tem sido amplamente comercializado e consumido no Brasil.

\section{Material e métodos}

\subsection{Obtenção dos exsudatos cárneos}

Para a avaliação da aplicabilidade da técnica de ELISA em exsudatos de carnes de sol, utilizamos 32 cortes comerciais de bovinos infectados experimentalmente (MARCIANO, 2013) (coxão duro - Bicepis femoris; coxão mole - Semi membranosus; contrafilé - Longissimus dorsi; paleta - Supra spinatus; alcatra - Gluteos medios; patinho - Quadricepis femoris; lagarto - Semi tendinosus; filé-mignon - Psoas major), sendo 22 provenientes de três bovinos inoculados com $5 \times 10^{2}$ cistos da cepa ME49 de T. gondii, via subcutânea (MEIRELES, 2005), e 10 carnes de sol confeccionadas com cortes de bovinos do grupo controle, inoculados com $1 \mathrm{~mL}$ de solução salina estéril. As carnes de sol, pesando cerca de $1.000 \mathrm{~g}$ cada, foram produzidas artesanalmente, em laboratório, com a adição de cloreto de sódio, segundo o protocolo descrito na literatura (COUTINHO, 2011).

As amostras foram congeladas overnight a $-17^{\circ} \mathrm{C}$ e, posteriormente, descongeladas por cerca de 4 horas, em temperatura ambiente, para obtenção dos exsudatos cárneos, de acordo com o protocolo preconizado por Mecca et al. (2011).

\subsection{Mensuração da hemoglobina presente nas amostras de carnes de sol}

Os exsudatos foram diluídos (1/10) em tampão contendo azida sódica (Tris/ $\mathrm{HCl}, \mathrm{NH}_{4} \mathrm{Cl}, \mathrm{NaN}_{3}$ ), o que permitiu a mensuração e a conversão da hemoglobina em um composto estável. Posteriormente, foram submetidos à leitura em espectrofotômetro digital, apropriado para leitura de microplacas de 96 poços. Esta leitura ocorreu nas amostras antes da adição do sal e após a salga, com o objetivo de verificar a interferência do sal na exsudação da carne. Após aferição, todos os exsudatos que apresentavam diferentes concentrações de sangue foram diluídos novamente em solução contendo azida sódica, após cálculo da quantidade ideal para que todas as amostras de exsudatos fossem ensaiadas em quantidades iguais de sangue, ou seja, em uma única diluição. A diluição do exsudato na DO 0,116 representa a quantidade de hemoglobina presente ideal, considerada como a concentração ideal de sangue, suficiente para a detecção de anticorpos específicos. Esta mensuração da quantidade de sangue no exsudato cárneo é extremamente importante para avaliar se a amostra pode ser ensaiada, pois, caso esteja muito diluída, pode gerar resultados falso negativos no ELISA (MARCIANO, 2013). Para as 42 amostras de carne de sol adquiridas no varejo (comerciais), a mensuração da quantidade de sangue foi realizada somente após o processamento, ou seja, após adição do sal.

\subsection{Ensaio imunoenzimático (ELISA)}

A detecção de IgG anti-T. gondii por ELISA foi realizada como descrito por Meireles et al. (2004). Amostras de exsudatos cárneos, previamente diluídas para uma concentração final de $0,116 \mathrm{~nm}$, foram aplicadas (100 $\mu \mathrm{L} /$ poço) em placas de 96 poços sensibilizadas com $10 \mu \mathrm{g} / \mathrm{mL}$ de extrato proteico de $T$. gondii e incubadas a $37^{\circ} \mathrm{C}$, por uma hora. Após este período, as placas foram lavadas três vezes com PBS contendo 0,05\% Tween 20 e, em seguida, foram adicionados $100 \mu \mathrm{L} /$ poço do conjugado anti-IgG bovino marcado com peroxidase $\left(\right.$ Sigma $\left.{ }^{\circledR}\right)$, sendo as placas então incubadas novamente a $37^{\circ} \mathrm{C}$, por uma hora. A reação foi revelada com OPD e interrompida com HCL 4M. O resultado do ELISA foi determinado pela absorbância (densidade óptica) a $492 \mathrm{~nm}$ em leitor automático de microplacas, sendo estabelecido um cut off de 0,470 nm, distinguindo as amostras positivas das negativas.

A análise estatística dos dados obtidos foi realizada pelos programas Graph Pad Prism 3.0 e Epilnfo 6.01.

\section{Resultados e discussão}

Vários estudos foram descritos utilizando amostras de exsudato cárneo (meat juice) para detecção de anticorpos por ELISA para doenças, cuja principal fonte de transmissão é a carne, como é o caso da triquinelose (NÖCKLER et al., 2005), da salmonelose (SZABÓ et al., 2008) e da cisticercose (ABUSEIR et al., 2007). Em relação à toxoplasmose, existem estudos que descrevem a utilização do exsudato cárneo na detecção de anticorpos por ELISA como um possível método alternativo ao soro para suínos (HILL et al., 2006; BERGER-SCHOCH et al., 2011), ovinos, javalis e bovinos (BERGER-SCHOCH et al., 2011) e para coelhos (MECCA et al., 2011).

Nossos resultados denotam a importância de se aferir a quantidade de sangue presente nos exsudatos, ajustando as amostras numa mesma concentração de sangue, por meio da diluição padronizada, principalmente nas carnes processadas, como a carne de sol, o que permite evitar, assim, resultados falsos positivos e/ou negativos nas amostras. A Figura 1 apresenta a interferência na exsudação das amostras de carnes $\left(n^{\circ}=32\right)$ obtidas de 


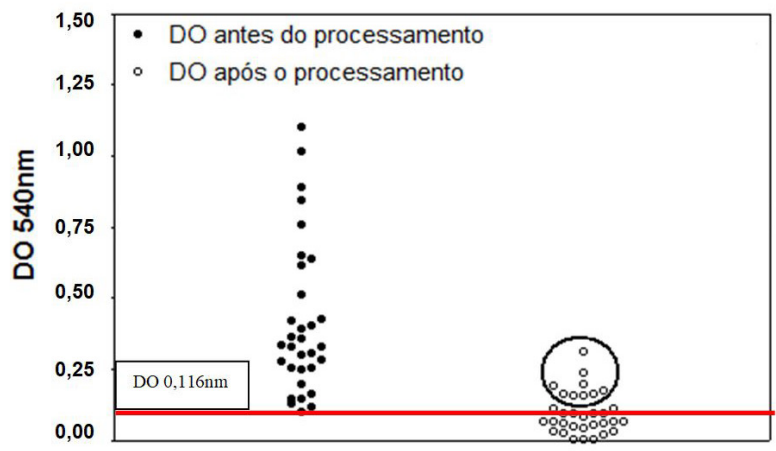

Amostras de carne de sol artesanal

Figura 1. Mensuração da DO $540 \mathrm{~nm}$ de amostras de carnes de sol $\left(n^{\circ}=32\right)$ antes e após o processamento dos cortes experimentais com adição de sal. O círculo representa as amostras com DO $540 \mathrm{~nm} \geq 0,116$, ou seja, as amostras que apresentaram sangue suficiente para ser ensaiadas mesmo após a adição do sal $\left(n^{\circ}=9\right)$.

animais experimentais antes e após a adição do sal, quando mensuradas na DO $540 \mathrm{~nm}$. Como pode ser observado, o valor das densidades ópticas sob filtro de $540 \mathrm{~nm}$ obtidas das amostras artesanais, após processamento pela adição de cloreto de sódio, sofreu um decréscimo acentuado em relação ao valor das mesmas amostras com leitura antes do processamento, quando todas as 32 amostras apresentaram quantidade suficiente de sangue para serem diluídas e ajustadas para uma absorbância padronizada $(0,116 \mathrm{~nm})$, para posterior ensaio. Assim, após a adição do sal, para obtenção da carne de sol artesanal, das 32 amostras analisadas, somente nove (28\%) apresentaram valor de DO $540 \mathrm{~nm}$ acima de 0,116 nm, sendo estas consideradas com quantidade de sangue suficiente para serem ensaiadas no ELISA.

Resultados semelhantes podem ser observados na Figura 2 para amostras de carnes de sol comerciais, em que, de um total de 42 amostras, somente seis (14,28\%) apresentaram quantidade de sangue suficiente (DO acima de $0,116 \mathrm{~nm}$ ), quando mensuradas na DO $540 \mathrm{~nm}$, podendo então ser ensaiadas pela ELISA.

A Figura 3 demonstra os resultados da ELISA para detecção de anticorpos IgG anti-T. gondii nas amostras de carnes de sol artesanal $(n=9)$ e comercial $(n=6)$ que obtiveram leitura igual ou superior a $0,116 \mathrm{~nm}$, quando submetidas à absorbância pela DO 540 nm, consideradas então com quantidade suficiente de sangue para serem ensaiadas. Como se pode observar, das seis amostras de carne de sol comercial, somente uma apresentou resultado positivo no ELISA, sendo condizente com a literatura, que relata baixa positividade de $T$. gondii em carnes bovinas, apesar da escassez de dados. Já para as amostras de carne de sol artesanal, processadas em laboratório, de nove amostras testadas, sete foram positivas e duas negativas (cut off de 0,470 nm). Estes resultados eram

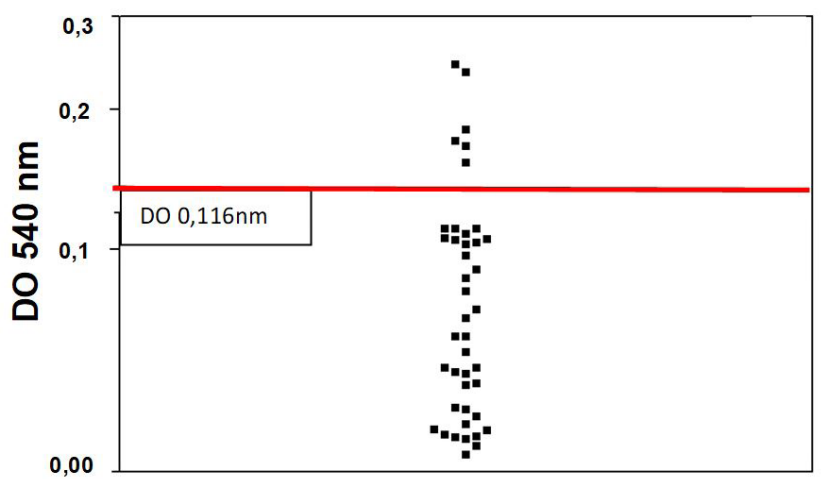

Amostras comerciais de carne de sol

Figura 2. Mensuração da DO 540 nm em amostras comerciais de carne de sol. Quantidade de exsudatos que não apresentaram quantidade suficiente de sangue, abaixo da linha vermelha $(0,116 \mathrm{~nm})$ de absorbância. Somente seis amostras apresentaram DO acima de 0,116 nm.

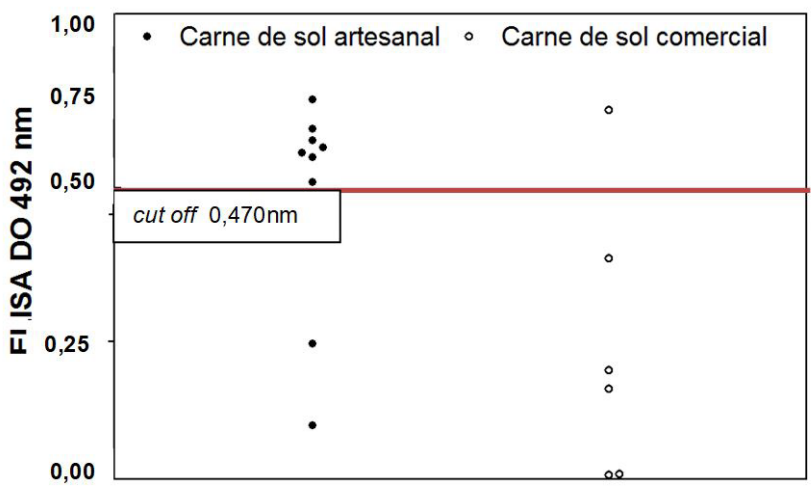

Amostras de carne de sol

Figura 3. Resultado do ELISA para detecção de anticorpos IgG anti-T. gondii em amostras de carnes de sol artesanal e comercial, cut off de $0,470 \mathrm{~nm}$, distinguindo as amostras positivas das negativas.

esperados, uma vez que as sete amostras positivas eram provenientes de cortes de carne de bezerros infectados com T. gondiie as duas amostras negativas eram provenientes de bezerros do grupo controle.

A maioria dos estudos relata a utilização de uma diluição comum para todas as amostras de exsudato, não levando em consideração a quantidade real de sangue nestas amostras. Isto pode resultar em uma medida subestimada, para amostras positivas altamente diluídas, ou uma medida superestimada, em amostras negativas, ensaiadas em altas concentrações, devido a reações inespecíficas, prejudicando a sensibilidade e a inespecificidade dos testes (DE LANGE et al., 2003; HILL et al., 2006; NIELSEN et al., 1998; NÖCKLER et al., 2004; WILHELM et al., 2007).

O ensaio imunoenzimático, proposto neste trabalho, utiliza uma quantidade fixa e padronizada de sangue para 
todas as amostras de exsudatos analisados, mensurando a quantidade de sangue pela leitura de densidade óptica (DO) em filtro de $540 \mathrm{~nm}$, garantindo que estas amostras tenham quantidade suficiente de imunoglobulinas para detecção de anticorpos IgG anti-T. gondii. Assim, se a amostra for negativa, pode-se afirmar que este resultado ocorreu porque a amostra realmente não apresenta IgG anti-T. gondii e não porque não há quantidade suficiente de sangue para detecção destes anticorpos (MECCA et al., 2011).

Nossos dados mostraram que o processamento da carne pela adição de cloreto de sódio interfere na exsudação da carne, limitando a utilização do exsudato cárneo como material para detecção de lgG anti-T. gondii em sistemas de monitoramento da qualidade de cortes comerciais de carnes processadas. Warnekulasuriya et al. (1998) já haviam demonstrado a interferência do sal na exsudação de carnes curadas e na detecção de micro-organismos pelo ensaio de PCR, em decorrência da alteração da pressão osmótica das células. Outro efeito que ocorre na carne de sol é a mudança de cor, já que, durante o processamento, a utilização de sais transforma a mioglobina (pigmento vermelho) em metamioglobina e ferro hemocromo, ambos de cor castanha (CHASCO et al., 1996). Essa transformação pode inibir a mensuração da densidade óptica a $540 \mathrm{~nm}$, que mensura a quantidade de sangue no exsudato pela presença da coloração vermelha da mioglobina. Assim, a utilização do ELISA para exsudatos de carnes processadas é limitada e pode ser aplicada somente quando o exsudato cárneo obtido apresentar quantidade suficiente de sangue para ser ensaiado.

\section{Conclusão}

Nossos resultados mostraram que é factível a utilização do exsudato cárneo para detecção de IgG anti-T. gondii, em cortes de carne bovina, pela técnica de ELISA, utilizando diluição única e padronizada, sendo esta uma ferramenta útil para o controle da qualidade de T. gondii em cortes comerciais de carne, devido a otimização, confiabilidade e reprodutibilidade dos testes (MECCA et al., 2011). Entretanto, para carnes de sol, nossos resultados demonstram que o processamento da carne pela adição de cloreto de sódio interfere na exsudação da carne, limitando a utilização do exsudato cárneo como material para detecção de IgG anti-T. gondii em carnes processadas, pois tal detecção depende da qualidade e quantidade do exsudato cárneo. Vale ressaltar a importância de avaliar outros métodos de obtenção do exsudato cárneo, como, por exemplo, o uso de solução salina e o maceramento do tecido muscular.

Neste contexto, a abordagem proposta em nosso trabalho constitui uma ferramenta bastante promissora para a rotina de Laboratórios de Saúde Pública, para monitoramento de carnes destinadas ao consumo humano. Também pode ser utilizada para elucidação de surtos epidêmicos envolvendo a carne bovina, abrindo perspectivas futuras de aplicação desta metodologia não só para a toxoplasmose, mas também para o controle de outras zoonoses transmitidas pela carne e de importância em Saúde Pública.

\section{Referências}

ABUSEIR, S.; KÜHNE, M.; SCHNIEDER, T.; KLEIN, G.; EPE, C. Evaluation of a serological method for the detection of Taenia saginata cysticercosis using serum and meat juice samples. Parasitology Research, v. 101, n. 1, p. 131-137, 2007. PMid:17216238. http://dx.doi.org/10.1007/s00436-006-0429-z.

BARIL, L.; ANCELLE, T.; GOULET, V.; THULLIEZ, P.; TIRARDFLEURY, V.; CARME, B. Risk factors for Toxoplasma infection in pregnancy: a case control study in France. Scandinavian Journal of Infectious Diseases, v. 31, n. 3, p. 305-309, 1999. PMid:10482062.

BERGER-SCHOCH, A. E.; BERNET, D.; DOHERR, M. G.; GOTTSTEIN, B.; FREY, C. F. Toxoplasma gondii in Switzerland: a serosurvey based on meat juice analysis of slaughtered pigs, wild boar, sheep and cattle. Zoonoses and Public Health, v. 58, n. 7, p. 472-478, 2011. PMid:21824348. http://dx.doi. org/10.1111/j.1863-2378.2011.01395.x.

CHASCO, J.; LIZASO, G.; BERIAIN, M. J. Cured colour development during sausage processing. Meat Science, v. 44, n. 3, p. 203-211, 1996. PMid:22060830. http://dx.doi.org/10.1016/ S0309-1740(96)00092-7

COOK, A. J.; GILBERT, R. E.; BUFFOLANO, W.; ZUFFEREY, J.; PETERSEN, E.; JENUM, P. A.; FOULON, W.; SEMPRINI, A. E.; DUNN, D. T. Sources of Toxoplasma infection in pregnant women: European multicentre case-control study. BMJ, v. 321, n. 7254, p. 142-147, 2000. PMid:10894691. http://dx.doi.org/10.1136/ bmj.321.7254.142.

COUTINHO, J. P. Produção e caracterização da carne de sol da carne de caprinos da raça anglo nubiana elaborada com diferentes teores de cloreto de sódio. 2011. 63 f. Dissertação (Mestrado em Engenharia de Processos de Alimentos)-Universidade Estadual do Sudoeste da Bahia, Itapetinga, 2011.

DE LANGE, K.; HADDAD, N.; LE POTIER, M. F.; LE VEE, M.; AMAR, P.; TOMA, B. Specificity of three ELISA-gE kits for screening pig meat for antibodies to Aujeszky's disease. The Veterinary Record, v. 153, n. 20, p. 621-624, 2003. PMid:14653341. http:// dx.doi.org/10.1136/vr.153.20.621.

DIAS, R. A. F.; FREIRE, R. L. Surtos de toxoplasmose em seres humanos e animais. Semina: Ciências Agrárias, v. 26, p. 239-248, 2005.

DUBEY, J. P. Survival of Toxoplasma gondii tissue cysts in $0.85-6 \% \mathrm{NaCl}$ solutions at $4-20^{\circ} \mathrm{C}$. The Journal of Parasitology, v. 83 , n. 5, p. 946-949, 1997. PMid:9379305. http://dx.doi. org/10.2307/3284295. 
Avaliação da técnica de ELISA para pesquisa de IgG anti-Toxoplasma gondii em exsudatos de carnes de sol Marciano, M. A. M. et al.

DUBEY, J. P.; KIRKBRIDE, C. A. Economic and public health considerations of congenital toxoplasmosis in lambs. Journal of the American Veterinary Medical Association, v. 195, n. 12, p. 1715-1716, 1989. PMid:2599957.

DUBEY, J. P.; KOTULA, A. W.; SHARAR, A. K.; ANDREWS, C. D.; LINDSAY, D. S. Effect of high temperature on infectivity of Toxoplasma gondii tissue cysts in pork. The Journal of Parasitology, v. 76, n. 2, p. 201-204, 1990. PMid:2319420. http://dx.doi.org/10.2307/3283016.

DUBEY, J. P.; THULLIEZ, P. Persistence of tissue cysts in edible tissues of cattle fed Toxoplasma gondii oocysts. American Journal of Veterinary Research, v. 54, n. 2, p. 270-273, 1993. PMid:8430937

FRENKEL, J. K.; DUBEY, J. P.; MILLER, N. L. Toxoplasma gondii in cats: fecal stages identified as coccidian oocysts. Science, v. 167, n. 3919, p. 893-896, 1970. PMid:4903651. http://dx.doi. org/10.1126/science.167.3919.893.

HILL, D. E.; CHIRUKANDOTH, S.; DUBEY, J. P.; LUNNEY, J. K.; GAMBLE, H. R. Comparison of detection methods for Toxoplasma gondii in naturally and experimentally infected swine. Veterinary Parasitology, v. 141, n. 1-2, p. 9-17, 2006. PMid:16815636. http://dx.doi.org/10.1016/j.vetpar.2006.05.008.

JAMRA, L. M.; MARTINS, M. C.; VIEIRA, M. P. Ação do sal de cozinha sobre o Toxoplasma gondii. Revista do Instituto de Medicina Tropical de São Paulo, v. 33, n. 5, p. 359-363, 1991. http://dx.doi.org/10.1590/S0036-46651991000500004.

KOTULA, A. W.; DUBEY, J. P.; SHARAR, A. K.; ANDREWS, C. D.; SHEN, S. K.; LINDSAY, D. S. Effect of freezing on infectivity of Toxoplasma gondiitissue cysts in pork. Journal of Food Protection, v. 54, n. 9, p. 687-690, 1991. http://dx.doi.org/10.4315/0362028X-54.9.687

MARCIANO, M. A. M. Pesquisa de IgG anti-Toxoplasma gondii em exsudato cárneo para monitoramento da qualidade da carne bovina. 2013. 104 f. Dissertação (Mestrado em Doenças Tropicais e Saúde Internacional)-Instituto de Medicina Tropical, Universidade de São Paulo, São Paulo, 2013.

MECCA, J. N.; MEIRELES, L. R.; ANDRADE JUNIOR, H. F. Quality control of Toxoplasma gondii in meat packages: standardization of an ELISA test and its use for detection in rabbit meat cuts. Meat Science, v. 88, n. 3, p. 584-589, 2011. PMid:21316867. http://dx.doi.org/10.1016/j.meatsci.2011.01.016.

MEIRELES, L. R. Padronização e aplicações da avidez de anticorpos IgG no diagnóstico laboratorial da toxoplasmose animal. 2005. 117 f. Tese (Doutorado)-Instituto de Ciências Biomédicas, Universidade de São Paulo, São Paulo, 2005.

MEIRELES, L. R.; GALISTEO JUNIOR, J. R.; POMPEU, E.; ANDRADE JUNIOR, H. F. Toxoplasma gondii spreading in an urban area evaluated by seroprevalence in free-living cats and dogs. Tropical Medicine \& International Health, v. 9, n. 8, p. 876-881, 2004. PMid:15303992. http://dx.doi.org/10.1111/j.13653156.2004.01280.x
MENUCCI, T. A.; MARCIANO, M. A. M.; ATUI, M. B. Avaliação da contaminação por matérias estranhas em carnes-de-sol comercializadas em casas do norte. Revista do Instituto Adolfo Lutz, v. 1, p. 47-54, 2010.

NARDI, G.; NARDI, K. F.; COLENCI, R.; SANTOS, E. L. B. Toxoplasmose: aspectos de saúde pública e importância ao agronegócio. Tékhne e Logos, v. 3, p. 1-19, 2012.

NAVARRO, I. T.; VIDOTTO, O.; GIRALDI, N.; MITSUKA, R. Resistência do Toxoplasma gondii ao cloreto de sódio e aos condimentos em linguiça de suínos. Boletin de la Oficina Sanitaria Panamericana, v. 112, n. 2, p. 138-143, 1992. PMid:1531110.

NIELSEN, B.; EKEROTH, L.; BAGER, F.; LIND, P. Use of muscle fluid as a source of antibodies for serologic detection of Salmonella infection in slaughter pig herds. Journal of Veterinary Diagnostic Investigation, v. 10, n. 2, p. 158-163, 1998. PMid:9576343. http:// dx.doi.org/10.1177/104063879801000207.

NÖCKLER, K.; HAMIDI, A.; FRIES, R.; HEIDRICH, J.; BECK, R.; MARINCULIC, A. Influence of methods for Trichinella detection in pigs from endemic and non-endemic European region. Journal of Veterinary Medicine. B, Infectious Diseases and Veterinary Public Health, v. 51, n. 6, p. 297-301, 2004. PMID: 15458494. http://dx.doi.org/10.1111/j.1439-0450.2004.00770.x.

NÖCKLER, K.; SERRANO, F. J.; BOIREAU, P.; KAPEL, C. M. O.; $\mathrm{POZIO}, \mathrm{E}$. Experimental studies in pigs on Trichiella detection in different diagnostic matrices. Veterinary Parasitology, v. 132, n. 1-2, p. 85-90, 2005. PMid:15985334. http://dx.doi.org/10.1016/j. vetpar.2005.05.033

SZABÓ, I.; SCHERER, K.; ROESLER, U.; APPEL, B.; NÖCKLER, K.; HENSEL, A. Comparative examination and validation of ELISA test systems for Salmonella typhimurium diagnosis of slaughtering pigs. International Journal of Food Microbiology, v. 124, n. 1, p. 65-69, 2008. PMid:18387686. http://dx.doi.org/10.1016/j. ijfoodmicro.2008.02.022.

TENTER, A. M. Toxoplasma gondii in animals used for human consumption. Memorias do Instituto Oswaldo Cruz, v. 104, n. 2, p. 364-369, 2009. PMid:19430665. http://dx.doi.org/10.1590/ S0074-02762009000200033.

VILLENA, I.; DURAND, B.; AUBERT, D.; BLAGA, R.; GEERS, R.; THOMAS, M.; PERRET, C.; ALLIOT, A.; ESCOTTE-BINET, S.; THÉBAULT, A.; BOIREAU, P.; HALOS, L. New strategy for the survey of Toxoplasma gondii in meat for human consumption. Veterinary Parasitology, v. 183, n. 3-4, p. 203-208, 2012. PMid:21864981. http://dx.doi.org/10.1016/j.vetpar.2011.08.001.

WARNEKULASURIYA, M. R.; JOHNSON, J. D.; HOLLIMAN, R. E. Detection of Toxoplasma gondii in cured meats. International Journal of Food Microbiology, v. 45, n. 3, p. 211-215, 1998. PMid:9926998. http://dx.doi.org/10.1016/S0168-1605(98)00158-5.

WILHELM, E.; HILBERT, F.; PAULSEN, P.; SMULDERS, F. J.; ROSSMANITH, W. Salmonella diagnosis in pig production: methodological problems in monitoring the prevalence in pigs and pork. Journal of Food Protection, v. 70, n. 5, p. 1246-1248, 2007. PMid:17536687. http://dx.doi.org/10.4315/0362-028X-70.5.1246. 